\title{
Hochwasserschutz in Deutschland
}

\section{Neue Modelle zur Abschätzung von Hochwasserschäden}

\author{
Die Hochwasserschäden der letzten Jahre haben \\ Fragen der Hochwasservorsorge wieder in den \\ öffentlichen Fokus gerückt. Um Hochwasserrisi- \\ ken zu quantifizieren, adäquate Schutzmaßnah- \\ men zu planen und hinsichtlich ihrer Effizienz \\ zu bewerten, sind Methoden zur Abschätzung \\ von Hochwasserschäden im gesamten Bundes- \\ gebiet notwendig. \\ Von Annegret Thieken
}

V erlässliche Abschätzungen von potenziellen Hochwasserschäden sind unerlässlich für Kosten-Nutzen-Untersuchungen bei der Planung von Hochwasserschutzmaßnahmen, für Prämienkalkulationen und Risikobetrachtungen in der Versicherungswirtschaft sowie für die Erstellung von Risikokarten. Da wenig über die Faktoren, die die Schäden beeinflussen können, bekannt ist, beschränken sich die meisten Schadensmodelle auf den Wasserstand als Eingangsgröße. Mit sogenannten Wasserstand-Schadensfunktionen werden potenzielle Schäden in verschiedenen Wirtschaftssektoren, beispielsweise in Privathaushalten, an öffentlichen Gebäuden oder im Dienstleistungssektor, abgeschätzt (MURL 2000; IKSR 2001).

In Deutschland basieren viele Schadensmodelle auf der Datenbank HOWAS, kurz für HOchWAsserSchäden. Sie enthält über 4000 Schadensfälle aus den Jahren 1978 bis 1994 mit einem Schwerpunkt in Süddeutschland (Buck/Merkel 1999). Die Schadenswerte und die aus HOWAS abgeleiteten WasserstandSchadensfunktionen weisen jedoch eine beträchtliche Streubreite auf, die vermutlich durch unterschiedliche, aber nicht erfasste Randbedingungen der Einzelschäden wie beispielsweise Vorsorgemaßnahmen verursacht werden (Merz et al. 2004). Da die HOWAS-Datenbank seit einigen Jahren nicht mehr fortgeführt wird, steht in Deutschland momentan keine aktuelle und konsistente Datengrundlage für Hochwasserschadensabschätzungen zur Verfügung.

Um die Unsicherheit von Schadensabschätzungen zu reduzieren, wurde das Forschungsprojekt Methoden zur Erfassung direkter und indirekter Hochwasserschäden (MEDIS) unter Leitung des Geoforschungszentrums Potsdam initiiert. Das Projekt ist Teil des Förderprogramms Risikomanagement extremer Hochwasserereignisse (RIMAX), welches vom Bundesministerium für Bildung und Forschung (BMBF) finanziert wird.

In MEDIS werden Methoden zur Erfassung und Abschätzung direkter und indirekter Schäden auf Basis aktueller Schadensda- ten neu entwickelt. Durch die Einbeziehung von weiteren Faktoren, neben dem Wasserstand, soll eine Verbesserung der herkömmlichen Modelle erreicht werden. Die Methoden sollen in einigen Testgebieten validiert und mit Wasserstands-Schadensfunktionen verglichen werden. Die Modellentwicklung erfolgt für verschiedene Schadenssektoren, und zwar für den privaten, gewerblichen, landwirtschaftlichen und öffentlichen Sektor. Ein Überblick über die wichtigsten Projektergebnisse wird in Thieken et al. (2008a) gegeben. Im vorliegenden Beitrag werden die Modellierungsansätze am Beispiel des privaten Sektors erläutert.

\section{Schadensdefinition}

Im Allgemeinen werden Hochwasserschäden in direkte und indirekte Schäden unterteilt. Direkte Schäden entstehen durch den physischen Kontakt mit dem Hochwasser, während indirekte Schäden zwar durch ein Hochwasser ausgelöst werden, aber räumlich oder zeitlich außerhalb des eigentlichen Ereignisses auftreten. Beide Schadensarten werden weiterhin in tangible und intangible Schäden unterteilt, je nachdem, ob sie monetär bewertet werden können oder nicht.

Im Projekt MEDIS wurde diese Schadensdefinition durch räumliche und zeitliche Aspekte erweitert (Abbildung 1). Durch die Hochwassereinwirkung können Objekte, zum Beispiel Gebäude, Straßen, Maschinen, Deiche oder Feldfrüchte, durch Verschmutzung oder Durchfeuchtung geschädigt oder zerstört werden. Darüber hinaus werden aber auch Schäden durch ansteigendes Grundwasser sowie langfristige Folgeschäden wie Schimmelbildung oder Frostschäden, die auf die physische Ein-

Abbildung 1: Auftreten tangibler direkter Hochwasserschäden in Raum und Zeit (der graue Bereich kennzeichnet den überfluteten Bereich/Zeitraum)

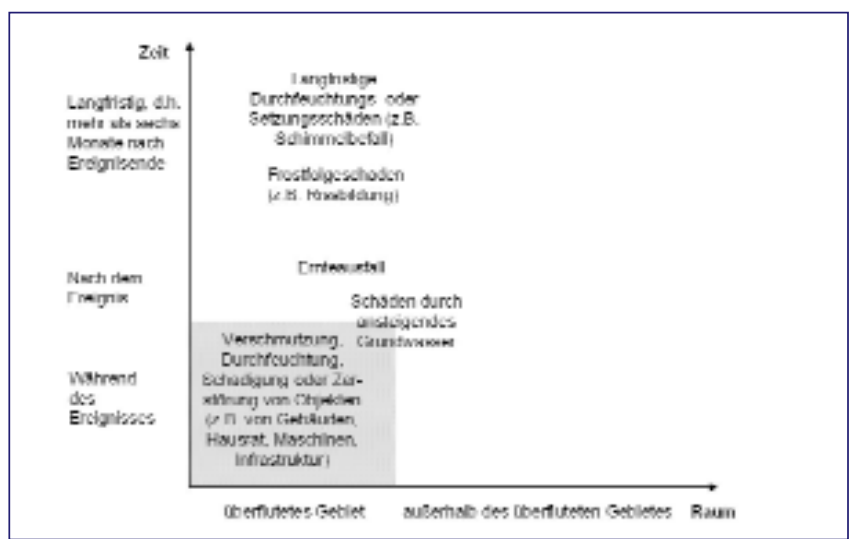

Quelle: Eigene Darstellung 
wirkung des Wassers zurückgeführt werden können, zu den direkten Hochwasserschäden gezählt, obwohl sie erst nach dem eigentlichen Schadensereignis sichtbar werden. Bei einer Schadenserfassung unmittelbar nach dem Ereignis werden diese direkten Folgeschäden oft noch nicht erkannt, können aber ein erhebliches Ausmaß erreichen.

Auch wenn der Fokus der Schadensabschätzung auf der Bewertung monetärer Schäden liegt, ist bei vielen Untersuchungen ein Hochwasserschaden nicht nur eine monetäre Größe. Gerade bei der Erstellung einer ersten Schadensbilanz wird der Schaden zunächst in physischen Einheiten, das heißt durch die Anzahl betroffener Personen und Gebäude oder anhand der Kilometer geschädigter Straßen, beziffert. Da der finanzielle Aufwand, um einen Schaden zu reparieren, auf Anhieb nur grob zu schätzen ist, können Einzelschäden zunächst qualitativ beschrieben und auf einer Ordinalskala klassifiziert werden, um das Schadensausmaß zu charakterisieren. So wurde im Rahmen des MEDIS-Projektes eine Klassifizierung struktureller Gebäudeschäden durch Schwarz und Maiwald (2007) entwickelt. Diese ist in Tabelle 1 dargestellt. Ein ähnliches Verfahren wurde für die Beschreibung von Schäden an Straßen angewendet.

Bei der Berechnung von monetären Schäden sind zwei Konzepte, das Brutto- und das Nettokonzept, zu unterscheiden. Während beim Bruttokonzept der Neuwert des geschädigten Objektes zugrunde gelegt wird, wird beim Nettokonzept der Zeitwert bewertet. Entsprechend wird im Bruttokonzept ein finanzieller Schaden durch die Wiederherstellungs- oder Wiederbeschaffungskosten angegeben. Beim Nettokonzept, das bei volkswirtschaftlichen Betrachtungen verwendet wird, muss die Wertsteigerung, die das Objekt durch die Reparatur erfahren hat, abgezogen werden. Bei der Schadensmodellierung wird häufig ein relativer Schaden errechnet, das bedeutet, der monetäre Schaden wird auf den Gesamtwert des geschädigten Objektes bezogen. Gerade bei solchen Auswertungen ist auf eine konsistente monetäre Bewertungsgrundlage zu achten.

\section{Schadensdaten}

Im Nachgang des August-Hochwassers 2002 wurden durch das Geoforschungszentrum Potsdam und die Deutsche Rückversicherung, Düsseldorf, in 1697 betroffenen Privathaushalten computergestützte Telefoninterviews durchgeführt (Kreibich et al. 2005; Thieken et al. 2005). Neben den direkten Schäden an Wohngebäuden und am Hausrat wurden zahlreiche Faktoren, die den Schaden beeinflussen könnten, abgefragt. Dazu gehören beispielsweise die Hochwassereinwirkung mit Wasserstand, Dauer und Fließgeschwindigkeit, die Kontamination des Flusswassers durch Abwasser, Öl oder andere Chemikalien, die Hochwasserwarnung und durchgeführte Notmaßnahmen, langfristige Vorsorgemaßnahmen sowie der Wert und die Eigenschaften des betroffenen Gebäudes sowie des Hausrats. Mit demselben Fragebogen wurden im November und Dezember 2006 Privathaushalte untersucht, die im Sommer 2005 oder im Frühjahr 2006 von Hochwasser betroffen waren. In beiden Kam- pagnen lag der räumliche Schwerpunkt der Befragungen im Elbe- und Donaueinzugsgebiet.

Abbildung 2 zeigt die durchschnittlichen Gebäudeschäden aus diesen Datensätzen für Bayern und Sachsen. Zum Vergleich wurden Schadensdaten aus der HOWAS-Datenbank von 1985 und 1988 sowie Daten der Sächsischen Aufbaubank (SAB) und einer Wohngebäudeversicherung herangezogen. Ausgewertet wurden die Gebäudeschäden als Wiederherstellungskosten. Dazu wurden in der HOWAS-Datenbank die Schäden an Gebäude und festem Inventar zusammengefasst. Aus der Fördermitteldatenbank der SAB wurden die förderfähigen Kosten als Gebäudeschaden interpretiert. Bei den Versicherungsdaten wurde zum Schadensaufwand der Selbstbehalt addiert. Außerdem wurden Fälle, in denen der Selbstbehalt unterschritten wurde oder aus anderen Gründen kein Schadensaufwand anfiel, nicht berücksichtigt. Alle Schadensangaben wurden mithilfe des Baupreisindexes auf Preise für das Jahr 2005 umgerechnet.

Wie Abbildung 2 zeigt, treten große Unterschiede zwischen den mittleren Wohngebäudeschäden in Bayern und Sachsen sowie Unterschiede zwischen den verschiedenen Ereignissen auf. So sind die Hochwasserschäden in Bayern 2002 und 2005 mehr als doppelt so hoch wie in den 1980er Jahren und belegen somit die Notwendigkeit, Schadensauswertungen und -funktionen laufend zu aktualisieren.

In Sachsen liegen die durchschnittlichen Schäden durchgängig über denen aus Bayern. Dies ist für 2002 vermutlich sowohl auf die Stärke des Ereignisses zurückzuführen, als auch auf die großzügigen staatlichen Aufbauhilfen. In den drei Datensätzen mit Schäden vom Hochwasser 2002 in Sachsen fallen die Schäden aus der Telefonbefragung am niedrigsten aus. Ein Grund dafür ist sicherlich, dass Telefonbefragungen methodisch bedingt keine Totalschäden enthalten. Die Größenordnung der Schäden ist dennoch ähnlich.

\section{Das multi-faktorielle Schadensmodell}

Aus den Befragungsdaten wurde für den privaten Bereich das multi-faktorielle Schadensmodell FLEMOps, kurz für Flood Loss Estimation Model for the Private Sector, abgeleitet. Es ist ein- $\rightarrow$

Tabelle 1: Unterscheidung struktureller Schadensgrade infolge Hochwassereinwirkung

\begin{tabular}{|c|c|c|}
\hline Schadensgrad & Schadensbeschreibung & Merkmale \\
\hline D1 & $\begin{array}{l}\text { kein struktureller } \\
\text { Schaden }\end{array}$ & reiner Durchfeuchtungsschaden, ... \\
\hline D2 & geringer Schaden & $\begin{array}{l}\text { geringfügige Risse, eingedrückte } \\
\text { Türen und Fenster, Austausch von } \\
\text { Bauteilen erforderlich, ... }\end{array}$ \\
\hline D3 & mittlerer Schaden & $\begin{array}{l}\text { größere Risse an tragenden Wän- } \\
\text { den, Unterspülungen der Funda- } \\
\text { mente, Setzungen, ... }\end{array}$ \\
\hline D4 & schwerer Schaden & $\begin{array}{l}\text { Einsturz von tragenden Wänden, } \\
\text { Decken , ... }\end{array}$ \\
\hline D5 & $\begin{array}{l}\text { sehr schwerer } \\
\text { Schaden }\end{array}$ & $\begin{array}{l}\text { Kollaps bzw. Einsturz von größeren } \\
\text { Gebäudeteilen , ... }\end{array}$ \\
\hline
\end{tabular}

Quelle: Schwarz/Maiwald 2007 
Abbildung 2: Durchschnittliche Gebäudeschäden in verschiedenen Datensätzen und Hochwasserereignissen (Bezugsjahr: 2005; n: Anzahl der Schadensfälle, Erläuterungen zu den Datensätzen im Text)

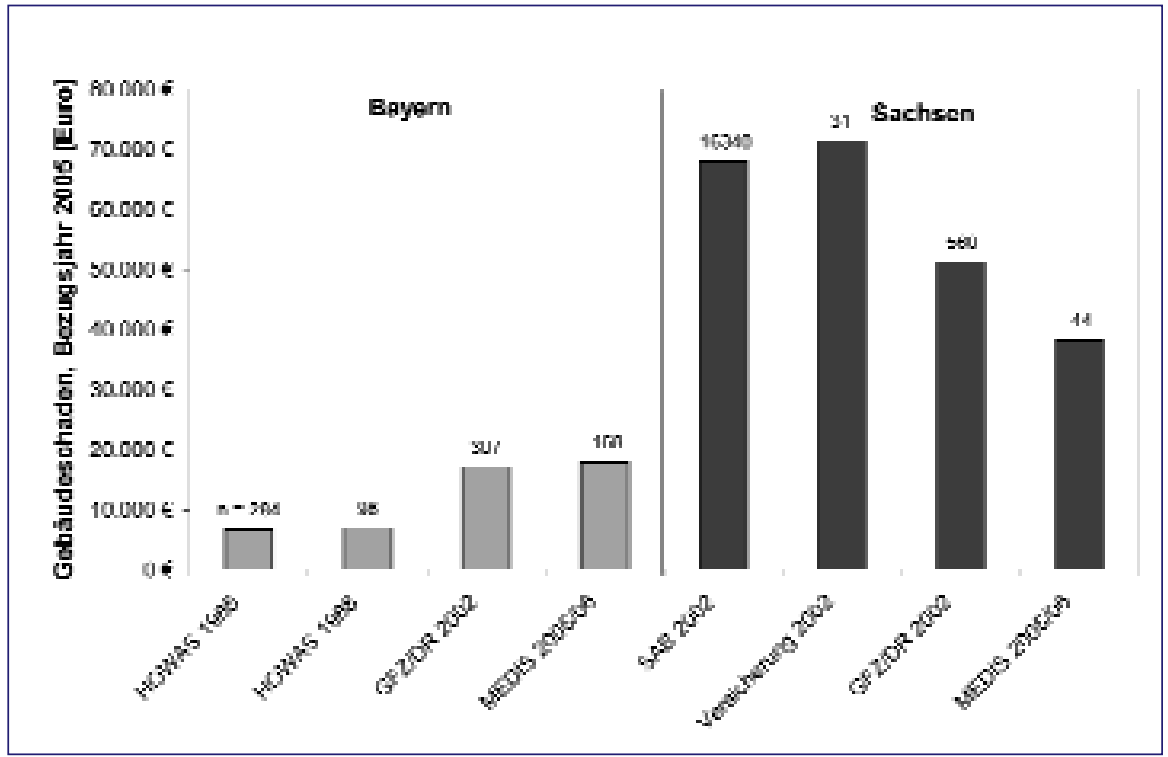

Quelle: Eigene Berechnungen

erseits auf der Mikroskala, also auf Ebene einzelner Gebäude, sowie andererseits auf der Mesoskala, das heißt auf Landnutzungseinheiten, anwendbar (Thieken et al. 2008b). Es berechnet den Schädigungsgrad oder den relativen Schaden getrennt für Wohngebäude und Hausrat. Das analoge Modell FLEMOcs, kurz für Flood Loss Estimation Model for the Commercial Sector, wurde für Schäden in Unternehmen, differenziert nach Schäden an Gebäuden, Betriebseinrichtungen sowie Waren, Produkten und Lagerbeständen, entwickelt (Kreibich et al. forthcoming).

Abbildung 3: Geschätzte Wohngebäudeschäden im Vergleich zu den dokumentierten förderfähigen Wiederherstellungskosten der Sächsischen Aufbaubank (SAB) in 13 Gemeinden in Sachsen für das Hochwasser 2002

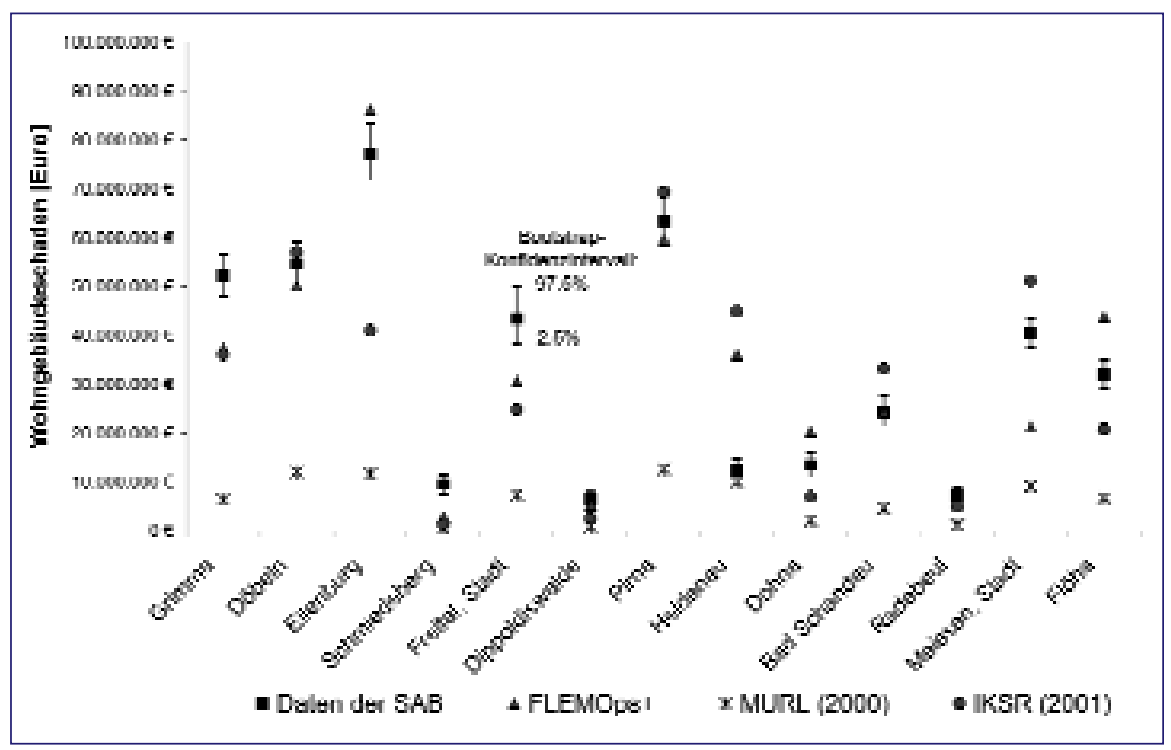

Quelle: Eigene Berechnungen
FLEMOps berücksichtigt in der ersten Modellstufe die Faktoren Wasserstand, Gebäudetyp und Gebäudequalität. Für die Ableitung des Scha- denmodells wurden die Einzelschäden aus der Befragung des Hochwassers 2002 klassifiziert, und zwar in fünf Wasserstandsklassen (bis 20 Zentimeter, 21 bis 60 Zentimeter, 61 bis 100 Zentimeter, 101 bis 150 Zentimeter, über 150 Zentimeter), drei Gebäudetypen (Einfamilienhaus, Reihen-/Doppelhaus, Mehrfamilienhaus) und zwei Gebäudequalitäts- oder Ausstattungsklassen (einfach bis mittel, gut bis sehr gut). Für alle Teildatensätze wurden mittlere Schädigungsgrade berechnet. Für die zweite Modellstufe, kurz FLEMOps+, wurden $\mathrm{Zu}$ - und Abschläge für verschiedene Ausprägungen der Kontamination des Flusswassers und der privaten Vorsorge aus den Daten abgeleitet

(Büchele et al. 2006).

Bei der Anwendung dieses mikroskaligen Schadenmodells wird für jedes betroffene Gebäude aus der Kombination aus Wasserstand, Gebäudetyp und Gebäudequalität ein mittlerer Schädigungsgrad abgeschätzt. Der berechnete relative Schaden kann in einer zweiten Stufe, falls die benötigten Informationen vorliegen, durch Multiplikation mit dem entsprechenden Faktor für Vorsorge und Kontamination angepasst werden. Um den absoluten Schaden zu erhalten, ist der Schädigungsgrad mit dem Gebäudewert zu multiplizieren.

Für mesoskalige Anwendungen ist das mikroskalige Schadenmodell so zu skalieren, dass es auf größere, einheitliche Landnutzungseinheiten anwendbar ist. In FLEMOps wurde diese Skalierung mit Hilfe von Marktforschungsdaten durchgeführt (Infas Geodaten 2001). Damit wurde für jede Gemeinde eine typische Gebäudezusammensetzung ermittelt. Die mittleren prozentualen Anteile der Gebäudetypen wurden dann verwendet, um die mikroskaligen Schadensmodelle zu gewichten. Auf diese Weise kann FLEMOps regional angepasst werden (Thieken et al. 2008b). Zusätzlich zur Skalierung des Schadensmodells wird der Vermögensbestand, der mithilfe von Angaben der statistischen Landesämter auf Gemeindeebene von Kleist et al. (2006) ermittelt wurde, mithilfe von Landnutzungsdaten disaggregiert. Ein 
mögliches Verfahren, auch „dasymteric mapping“ genannt, wird in Thieken et al. (2006) beschrieben.

Bei der mesoskaligen Schadensabschätzung wird pro Rasterzelle je nach Gemeinde und Wasserstandsklasse ein mittlerer Schädigungsgrad berechnet, der multipliziert mit dem zugehörigen Vermögensbestand einen absoluten Schaden pro Rasterzelle liefert. Wie beim mikroskaligen Modell kann der geschätzte Schaden mithilfe der Zu- und Abschläge für Kontamination und Vorsorge weiter regional korrigiert werden. Aufgrund der Ungenauigkeit der Eingangsdaten werden die geschätzten Schäden pro Gemeinde aufsummiert. Dieses Vorgehen erlaubt prinzipiell die deutschlandweite Anwendung des Schadenmodells.

\section{Modellvalidierung}

FLEMOps wurde mithilfe von Daten aus der Förderdatenbank der Sächsischen Aufbaubank (SAB) validiert. Die Modellierung wurde auf Basis der Überflutungsflächen aus Grabbert (2006) auf der Mesoskala durchgeführt. Das Ausmaß der privaten Vorsorge und der Kontamination des Flusswassers wurde mithilfe der Telefonbefragung 2002 pro Gemeinde eingeschätzt. Die Ergebnisse der Schadensabschätzung sind in Abbildung 3 dargestellt, die Fehlerstatistik in Tabelle 2. Zum Vergleich wurden die Schäden ebenfalls mit den Wasserstands-Schadensfunktionen aus dem Rheinatlas und einer Studie zu potenziellen Schäden am Rhein in Nordrhein-Westfalen des Ministeriums für Umwelt und Naturschutz, Landwirtschaft und Verbraucherschutz (MURL) geschätzt (IKSR 2001; MURL, 2000).

Um die Güte der Modellergebnisse einzuschätzen, wurde aus den Schadensdaten der SAB mithilfe eines ResamplingVerfahrens ein Konfidenzintervall abgeleitet, das ebenfalls in Abbildung 3 eingezeichnet ist. Insgesamt entstehen mit allen drei Modellen große Schätzfehler. Abbildung 3 zeigt, dass das MURL-Modell die Schäden bei Weitem unterschätzt. Das IKSR-Modell sowie das in MEDIS entwickelte Modell FLEMOps+ decken hingegen die Spannweite der aufgetretenen Schäden besser ab. Laut Fehlerstatistik in Tabelle 2 liefert das Modell FLEMOps+ die besten Schätzergebnisse. Allerdings liegen auch hier nur wenige Schätzwerte innerhalb des Bootstrap-Konfidenzintervalls.

Ein Vorteil des neuen Modells FLEMOps ist, dass die Schadensfunktionen auf die örtliche Gebäudezusammensetzung angepasst werden können. Allerdings ist zu bedenken, dass sowohl das IKSR-Modell als auch das MURL-Modell für das Rheineinzugsgebiet entwickelt wurden. Zur Einschätzung der regiona(Bezugsjahr: 2000)
Tabelle 2: Deskriptive Statistik der dokumentierten förderfähigen Wiederherstellungskosten der Sächsischen Aufbaubank (SAB) und der geschätzten Wohngebäudeschäden in 13 Gemeinden in Sachsen für das Hochwasser 2002

\begin{tabular}{lccrr}
\hline & $\begin{array}{l}\text { Förder- } \\
\text { fähige } \\
\text { Kosten } \\
\text { (SAB) }\end{array}$ & $\begin{array}{l}\text { MURL } \\
(\mathbf{2 0 0 0 )}\end{array}$ & $\begin{array}{l}\text { ICPR } \\
(\mathbf{2 0 0 1 )}\end{array}$ & $\begin{array}{l}\text { FLE- } \\
\text { MOps+ }\end{array}$ \\
\hline AnzahI Gemeinden & 13 & 13 & 13 & 13 \\
\hline Mittelwert (Millionen Euro) & 34,26 & 6,62 & 30,32 & 32,51 \\
\hline Median (Millionen Euro) & 32,02 & 6,78 & 33,20 & 30,62 \\
\hline Minimum (Millionen Euro) & 7,40 & 0,50 & 1,36 & 2,80 \\
\hline Maximum (Millionen Euro) & 76,88 & 12,74 & 69,29 & 86,14 \\
\hline Standardabweichung (Millionen Euro) & 24,09 & 4,43 & 22,28 & 23,81 \\
\hline
\end{tabular}

Quelle: Eigene Berechnungen

len Übertragbarkeit von Modellen sind weitere Validierungen notwendig.

\section{Beispielanwendung}

Das Modell FLEMOps wurde flächendeckend auf die Hochwassergefahrenkarten in Sachsen, die von der Landestalsperrenverwaltung Sachsen zur Verfügung gestellt wurden, angewendet. Abbildung 4 zeigt die überfluteten Gebiete sowie den Wohngebäudeschaden pro Einwohner und Gemeinde. Durch die Normierung des Schadens mit der Einwohnerzahl können die Gemeinden untereinander besser verglichen werden. Dennoch weisen, wie bei den absoluten Schäden, die größeren Städte wie Dresden oder Leipzig die höchsten Pro-Kopf-Schadenswerte auf.

\section{Ausblick}

Die hier vorgestellten Ansätze zur Schadensklassifikation, Datenerhebung, Modellentwicklung und -validierung stellen $\rightarrow$

Abbildung 4: Überflutungsflächen in Sachsen für Hochwasser mit einer mittleren Wiederkehrperiode von 200-300 Jahren sowie Gebäudeschäden pro Einwohner und Gemeinde, berechnet mit FLEMOps

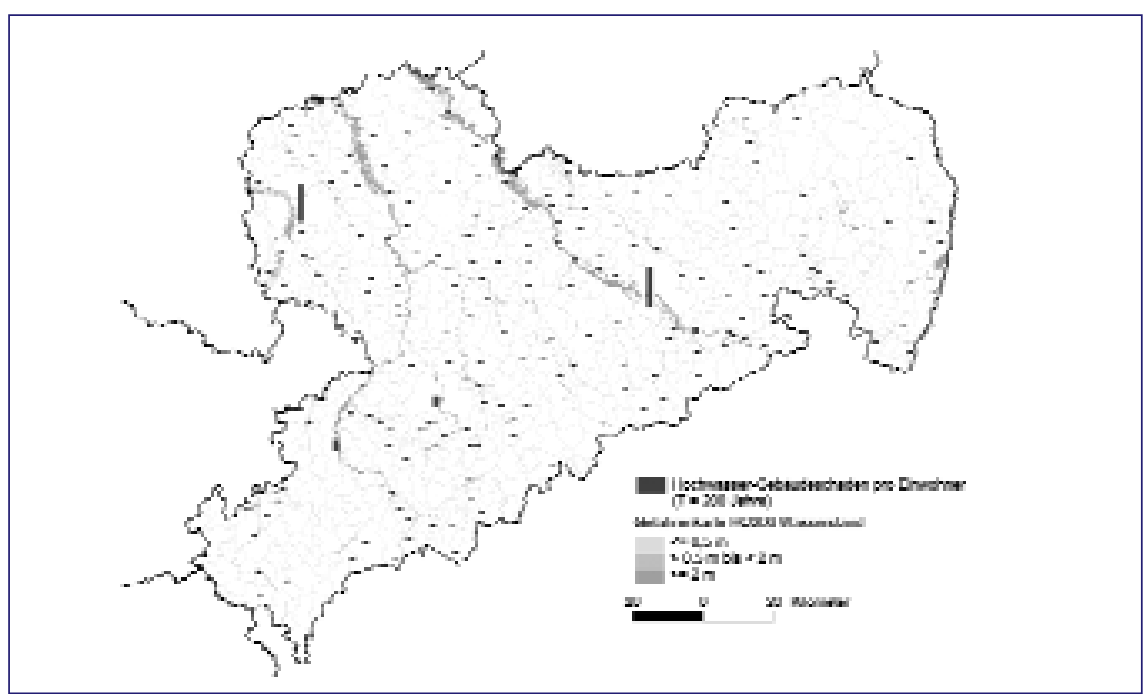

Quelle: Landestalsperrenverwaltung Sachsen und eigene Berechnungen 
Neuentwicklungen für die Hochwasserrisikoanalyse dar. Dies gilt insbesondere für die mikroskaligen Schadensfunktionen als auch die Skalierung von der Mikro- auf die Mesoskala, die auf empirischen Daten beruhen und transparent dargelegt werden. Bei ersten Modellvalidierungen in Sachsen wurde die offizielle Schadensangabe der Sächsischen Aufbaubank am besten mit dem neuen Modell FLEMOps unter Berücksichtigung von Kontamination und Vorsorge geschätzt. Es schneidet damit besser $\mathrm{ab}$ als andere, in Deutschland übliche mesoskalige Modelle. Weitere Modellanwendungen in mehreren Gemeinden und unter verschiedenen Hochwassereinwirkungen sind durchzuführen, bevor die Qualität des neuen Modells abschließend eingeschätzt werden kann.

\section{Anmerkung}

Die Telefonbefragung nach dem Hochwasser 2002 wurde als Kooperation zwischen dem Geoforschungszentrum Potsdam und der Deutschen Rückversicherung AG, Düsseldorf, durchgeführt. Die weiteren Ergebnisse wurden im Rahmen des Projektes Methoden zur Erfassung direkter und indirekter Hochwasserschäden (MEDIS) erarbeitet. Wir danken dem Bundesministerium für Bildung und Forschung (Förderkennziffer 0330688) und der Deutschen Rückversicherung AG für die finanzielle Unterstützung sowie der Sächsischen Aufbaubank und der Landestalsperrenverwaltung Sachsen für die Datenbereitstellung.

\section{Literatur}

Büchele, B. / Kreibich, H. / Kron, A. / Thieken, A.H. / Ihringer, J. / Oberle, P. / Merz, B. / Nestmann, F.: Flood-risk mapping: contributions towards an enhanced assessment of extreme events and associated risks. In: NHESS 6/2006, S. 485-503.

Buck, W. / Merkel, U.: Auswertung der HOWAS-Schadendatenbank. Karlsruhe 1999.

Grabbert, J.-H.: Analyse der schadensbeeinflussenden Faktoren des Hochwassers 2002 und Ableitung eines mesoskaligen Abschätzungsmodells für Wohngebäudeschäden. Universität Potsdam 2006.

IKSR (Internationale Kommission zum Schutz des Rheins): Rhein-Atlas. Koblenz 2001.

INFAS Geodaten: Das Data Wherehouse. Bonn 2001.
Kleist, L. / Thieken, A.H. / Köhler, P. / Müller, M. / Seifert, I. / Borst, D./ Werner, U.: Estimation of the regional stock of residential buildings as a basis for comparative risk assessment for Germany. In: NHESS 6, 4/2006, S. 541-552.

Kreibich H. / Thieken, A.H. / Petrow, T. / Müller, M. / Merz, B.: Flood loss reduction of private households due to building retrofitting - Lessons learned from the Elbe floods in August 2002. In: NHESS 5, 2005, S. 117-126.

Kreibich, H. / Seifert, I. / Merz, B. / Thieken, A.H.: FLEMOcs - A new model for the estimation of flood losses in companies. In: Hydrological Sciences Journal. Forthcoming.

Merz, B. / Kreibich, H. / Thieken, A. / Schmidtke, R.: Estimation uncertainty of direct monetary flood damage to buildings. In: NHESS 4, 1/2004, S. 153-163.

MURL (Ministerium für Umwelt, Raumordnung und Landwirtschaft des Landes Nordrhein-Westfalen): Potentielle Hochwasserschäden am Rhein in NRW. Düsseldorf 2000.

Schwarz, J. / H. Maiwald: Prognose der Bauwerksschädigung unter Hochwassereinwirkung. In: Bautechnik 84, 7/2007, S.450-464.

Thieken, A. et al.: Methods for the Evaluation of direct and indirect flood losses. Proceedings of the 4th International Symposium on Flood Defense, Toronto 2008a. S. 98-1-98-10 (CD-ROM).

Thieken, A.H. / Müller, M. / Kleist, L. / Seifert, I. / Borst, D. / Werner, U.: Regionalisation of asset values for risk analyses. In: NHESS 6, 2/2006, S. 167-178.

Thieken, A.H. / Müller, M. / Kreibich, H. / Merz, B.: Flood damage and influencing factors: New insights from the August 2002 flood in Germany. In: Water Resour. Res. 41, 12/2005 W12430.

Thieken, A.H. / Olschewski, A. / Kreibich, H. / Kobsch, S. / Merz, B.: Development and evaluation of FLEMOps - a new Flood Loss Estimation MOdel for the private sector. FRIAR Conference, WIT Press, London 2008b, S. 315-324.

\section{IAUTORIN + KONTAKT}

Annegret $\mathrm{H}$. Thieken leitet das Projekt MEDIS und ist Professorin für Naturgefahren- und Risikomanagement an der Leopold-Franzens-Universität Innsbruck sowie wissenschaftliche Leiterin von alpS.

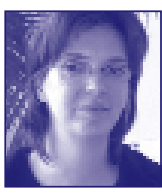

alpS - Zentrum für Naturgefahren- und Risikomanagement $\mathrm{GmbH}$, Grabenweg 3, 6020 Innsbruck, Österreich. Tel.: +43 51239292926 ,

E-Mail: thieken@alps-gmbh.com, annegret.thieken@uibk.ac.at

\section{oekom verlag}

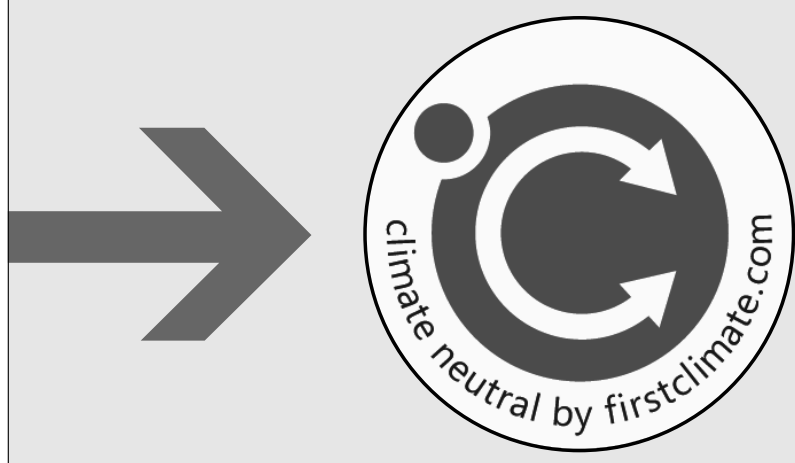

\section{Unser Ideal heißt klimaneutral}

Wir folgen dem Motto "Vom Wissen zum Handeln" und publizieren nicht nur zu Nachhaltigkeit, sondern leisten auch als Unternehmen einen aktiven Beitrag zum Klimaschutz: Seit Mai 2008 ist oekom der erste klimaneutrale Verlag Deutschlands.

Hierfür haben wir zunächst unseren $\mathrm{CO}_{2}$-Ausstoß in allen Betriebsabläufen so weit wie möglich reduziert - etwa bei der Wahl unseres Stromanbieters im Verlagssitz und in der Druckerei. Den unvermeidbaren Rest an $\mathrm{CO}_{2}$-Emissionen gleichen wir über unseren Partner First Climate AG aus: durch eine finanzielle Beteiligung an acht neuen Windkraftturbinen in Indien, bewertet nach Gold-Standard des World Wide Fund for Nature.

Die guten Seiten der Zukunft 
(c) 20I0 Authors; licensee IÖW and oekom verlag. This is an article distributed under the terms of the Creative Commons Attribution Non-Commercial No Derivates License (http://creativecommons.org/licenses/by-nc-nd/3.o/), which permits unrestricted use, distribution, and reproduction in any medium, provided the original work is properly cited. 